\title{
Erratum: Self-Diffusion for Particles with Stochastic Collisions in One Dimension ${ }^{1}$
}

\author{
C. Kipnis, J. L. Lebowitz, E. Presutti, and H. Spohn
}

Received February 2, 1983

The attention of the reader is drawn to the following misprints which appeared in the above reference paper:

Page 113, the second equality in equation (3.6) should be a plus sign, while the second equality in equation (3.7) should be a minus sign.

Page 114, Equation (3.9) should read:

$$
+2 \sum_{i=0}^{2} P\left(\sup _{0 \leqslant s \leqslant t} \sup _{\left|k-\rho \epsilon^{-2} s\right|<\lambda \epsilon^{-1} \sqrt{s}}\left|\epsilon \sum_{p=\rho \epsilon^{-2} s}^{k} v_{p-1}^{i} U_{p}^{i}\right| \geqslant \delta\right)
$$

\footnotetext{
${ }^{1}$ This paper appeared in J. Stat. Phys. 30:107 (1983).
} 